\title{
Buttressing Effects on Haloarene Deprotonation: A Merely Kinetic or also Thermodynamic Phenomenon?
}

\author{
Joanna Gorecka, Christophe Heiss, Rosario Scopelliti and Manfred Schlosser*
}

\section{Supporting Information}

Laboratory routine and abbreviations have been specified in previous publications. ${ }^{1-3}$ ${ }^{1} \mathrm{H}$ and $\left({ }^{1} \mathrm{H}\right.$ decoupled $){ }^{13} \mathrm{C}$ NMR were recorded at 400 and $101 \mathrm{MHz}$, respectively, the solvent being deuterochloroform. The X-ray crystallographic data have been deposited with the Cambridge Crystallographic Data Center, 12 Union Road, Cambridge CB2 1EZ, UK, from where they can be obtained free of charge at www.ccdc.cam.ac.uk/conts/retrieving. html.

\section{Working Procedure:}

At $-100{ }^{\circ} \mathrm{C}$, biphenyl (approx. $0.3 \mathrm{~g}, 2 \mathrm{mmol}$ ), as an internal reference ("standard") for gas chromatography quantification, (2,6-dichloro-4-iodophenyl)triethylsilane ${ }^{4}$ (1.9 g, 5.0 mmol) and, 15 min later, (2,6-dichloro-3-iodophenyl)triethylsilane were added consecutively to a solution of tert-butyllithium $(10 \mathrm{mmol})$ in tetrahydrofuran $(25 \mathrm{~mL})$ and pentanes $(7 \mathrm{~mL})$. Aliquots (of approx. $2.0 \mathrm{~mL}$ ) were withdrawn after 3, 15, 45, 180 and $360 \mathrm{~min}$ to be poured into a solution of elemental bromine (approx. $0.05 \mathrm{~mL}, 1 \mathrm{mmol}$ ) in hexanes $(3 \mathrm{~mL})$. The organic phase was washed with a saturated aqueous solution $(1.0 \mathrm{~mL})$ of sodium sulfite before being subjected to gas chromatographic analysis $\left(30 \mathrm{~m}\right.$, methylpolysiloxane $\mathrm{DB}-1,180{ }^{\circ} \mathrm{C} ; 30$

$\mathrm{m}$, polyethylene DB-WAX, $180{ }^{\circ} \mathrm{C}$ ). The amounts of iodo and bromo compounds ${ }^{5,6}$ were calculated from the product/standard ratios and corrected using separately determined calibration factors. 
(2,6-Dichloro-4-iodophenyl)triethylsilane: (2,6-Dichlorophenyl)triethylsilane (22 mL, 26 g, $0.10 \mathrm{~mol})$ was added to a solution of $\mathrm{sec}$-butyllithium $(0.10 \mathrm{~mol})$ in cyclohexane $(80 \mathrm{~mL})$ and tetrahydrofuran $(0.12 \mathrm{~L}) \mathrm{kept}$ in a dry ice bath. After $45 \mathrm{~min}$ at $-75^{\circ} \mathrm{C}$, the reaction mixture was poured into a solution of elemental iodine $(25 \mathrm{~g}, 0.10 \mathrm{~mol})$ in tetrahydrofuran $(0.10 \mathrm{~L})$ at $-75{ }^{\circ} \mathrm{C}$. The reaction mixture was washed with a saturated aqueous solution $(3 \mathrm{x} 25 \mathrm{~mL})$ of sodium sulfite. Upon distillation, a colorless oil was collected; b.p. $118-120^{\circ} \mathrm{C} / 0.2 \mathrm{mmHg}$; $n_{\mathrm{D}}^{20}=1.5962 ; d_{4}^{20}=1.429$; yield: $21.3 \mathrm{~g}(55 \%) ;{ }^{1} \mathrm{H}$ NMR: $7.60(\mathrm{~s}, 2 \mathrm{H}), 1.1(\mathrm{~m}, 6 \mathrm{H}), 1.0$ (m, 9 H). $-{ }^{13} \mathrm{C}$ NMR: $142.5,136.8,134.7,94.6,7.8$ (3 C), 6.0 (3 C). $-\mathrm{C}_{12} \mathrm{H}_{17} \mathrm{Cl}_{2} \mathrm{ISi}(387.15)$ : calcd. C 37.22, H 4.43; found C 37.44, H 4.30.

(2,6-Dichloro-3-iodophenyl)triethylsilane: Diisopropylamine $(14 \mathrm{~mL}, 10 \mathrm{~g}, 0.10 \mathrm{~mol})$ and 1-iodo-2,4-dichlorobenzene (13 mL, $27 \mathrm{~g}, 0.10 \mathrm{~mol})$ were added consecutively to a solution of butyllithium $(0.10 \mathrm{~mol})$ in hexanes $(65 \mathrm{~mL})$ and tetrahydrofuran $(0.15 \mathrm{~L})$ cooled in a dry ice/methanol bath. After $2 \mathrm{~h}$ at $-75^{\circ} \mathrm{C}$, the mixture was treated with chlorotriethylsilane $(18$ $\mathrm{mL}, 16 \mathrm{~g}, 0.10 \mathrm{~mol})$. Direct distillation afforded a colorless oil; b.p. $121-123{ }^{\circ} \mathrm{C} / 0.3 \mathrm{mmHg}$; $n_{\mathrm{D}}^{20}=1.5954 ; d_{4}^{20}=1.440$; yield: $32.5 \mathrm{~g}(84 \%) .-{ }^{1} \mathrm{H}$ NMR: $7.75(\mathrm{~d}, J=8.5 \mathrm{~Hz}, 1 \mathrm{H}), 6.93$ (d, $J=8.5 \mathrm{~Hz}, 1 \mathrm{H}), 1.1$ (m, $6 \mathrm{H}), 1.0$ (m, $9 \mathrm{H}) .-{ }^{13}$ C NMR: 145.0, 142.3, 141.3, 137.1, 129.8, 98.9, 7.9 (3 C), 6.3 (3 C). - $\mathrm{C}_{12} \mathrm{H}_{17} \mathrm{Cl}_{2} \mathrm{ISi}$ (387.15): calcd. C 37.23, $\mathrm{H}$ 4.43; found $\mathrm{C} 37.31, \mathrm{H}$ 4.36 .

(3-Bromo-2,6-dichlorophenyl)triethylsilane: Prepared as described in the preceding paragraph, from 1-bromo-2,4-dichlorobenzene ( $23 \mathrm{~g}, 0.10 \mathrm{~mol})$; colorless oil; b.p. 115 - 117 ${ }^{\circ} \mathrm{C} / 0.6 \mathrm{mmHg} ; n_{\mathrm{D}}^{20}=1.5660 ; d_{4}^{20}=1.325$; yield: $29.6 \mathrm{~g}(87 \%) .-{ }^{1} \mathrm{H}$ NMR: $7.51(\mathrm{~d}, J=8.5$ $\mathrm{Hz}, 1 \mathrm{H}), 7.09$ (d, $J=8.5 \mathrm{~Hz}, 1 \mathrm{H}), 1.1(\mathrm{~m}, 6 \mathrm{H}), 1.0(\mathrm{~m}, 9 \mathrm{H}) .-{ }^{13} \mathrm{C}$ NMR: 141.6, 138.4, 135.2, 130.2, 129.1, 123.2, 8.3 (3 C), 6.8 (3 C). $\mathrm{C}_{12} \mathrm{H}_{17} \mathrm{BrCl}_{2} \mathrm{Si}$ (340.15): calcd. C 42.37, H 5.04; found C 42.36, H 5.16. 


\section{References}

[1] Bobbio, C.; Schlosser, M.; Eur. J. Org. Chem. 2001, 4533 - 4536.

[2] Schlosser, M.; Heiss, C.; Eur. J. Org. Chem. 2003, 4618 - 4624.

[3] Schlosser, M.; Marull, M.; Eur. J. Org. Chem. 2003, 1569 - 1575.

[4] Prepared from the commercial 2,4-dichloro-iodobenzene and 1,3-dichloro-5iodobenzene by simultaneous treatment with lithium 2,2,6,6-tetramethylpiperidide (LITMP) and chlorotriethylsilane in tetrahydrofuran at $-75^{\circ} \mathrm{C}$ ("in situ trapping") followed by immediate distillation.

[5] Prepared from the commercial 1-bromo-2,4-dichlorobenzene and 1-bromo-3,5dichlorobenzene, respectively, as described in the preceding footnote for the $(2,6-$ dichloro-3- and -4-iodophenyl)triethylsilanes.

[6] C. Heiss, E. Marzi, M. Schlosser, Eur. J. Org. Chem. 2003, 4625 - 4629. 